\title{
TIME-DOMAIN SIGNAL MANAGEMENT FOR OFDM SIGNALS
}

\author{
Takuya Kazama $^{1}$, Kazuki Miyazawa ${ }^{2}$, and Masahiro Muraguchi ${ }^{3}$ \\ ${ }^{1,2}$ Faculty of Engineering, Tokyo University of Science, Tokyo, Japan \\ ${ }^{3}$ Tokyo University of Science, Tokyo, Japan
}

\begin{abstract}
We have found out that the CAZAC-OFDM accords the amplitude of IFFT output signal with the amplitude of input DATA and the time ordering of IFFT output signal is unambiguously determined. That is, the OFDM time-domain signals, which are composed of many sinewaves, can be shaped by CAZAC precoder. As one application example that can use this characteristic of CAZAC precoder, we propose a new technique of symbol timing estimation, which enable to avoid the use of the preambles and guard-intervals. Conventional OFDM systems introduce guard-intervals for symbol timing estimation and reduction of multipath effect. Visible light communications (VLCs), which are one kind of line-of-sight communications, does not require consideration of the multipath channel. Therefore, if we embed null data at a fixed position in time-domain, we will easily estimate the symbol timing in the receiver side.
\end{abstract}

\section{KEYWORDS}

OFDM; CAZAC sequence; Zadoff-Chu sequence; Symbol Timing Estimation; VLC.

\section{INTRODUCTION}

In recent years, with the development of wireless communication technology, the usage of wireless communication by smart phones, tablets, laptops, etc. has been increasing steadily. Meanwhile radio frequency bands are becoming exhausted, and Visible Light Communication (VLC) having much shorter wavelength than radio waves is being studied. Among visible light communication, studies using light emitting diodes (LED) become increasingly active [1], [2]. The reason for this is that high-speed communication due to the development of high-speed responsiveness of LEDs has become possible [3], and ease of dissemination due to the proliferation of illumination using LEDs also in general household.

A major drawback of VLC is band-limited operation due to the frequency characteristics of LEDs. Therefore, VLC requires a modulation scheme with high spectral efficiency. Orthogonal frequency division multiplexing (OFDM) scheme is very suitable for the VLC, because its great advantage of spectrum utilization that is about twice spectral efficiency compared with single carrier modulation scheme.

The OFDM system, however, requires highly exact timing for the symbol synchronization. The symbol timing shift in the time-domain on the receiver incurs a serious distortion after demodulation.

Therefore, conventional OFDM systems introduce preambles and guard-intervals for symbol timing estimation. Although the preamble enables high-precision symbol synchronization, it is 
only applied intermittently in a communication in which the beginning of a signal does not exist, for example, a signal continuously transmitted such as a broadcast. Even though the guard interval enables synchronization for each symbol, a significant throughput reduction is drawn, and it is wasteful considering the application in the VLC which is a line-of-sight wireless system anddoes not need to consider the multipath signals.
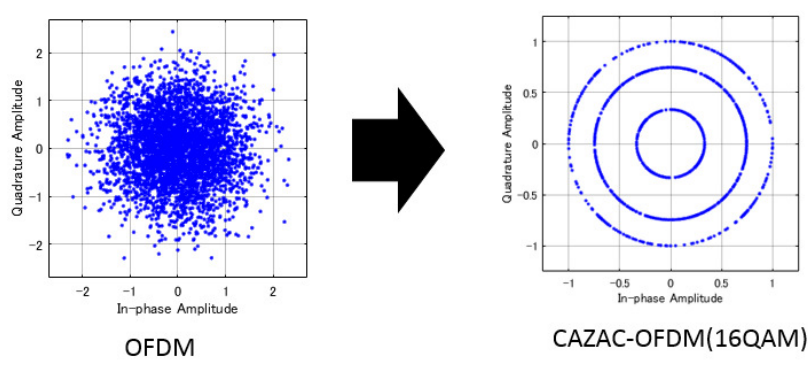

CAZAC-OFDM(16QAM)

Figure1.Time domain Signal without and with CAZAC precoder

Another well-known drawback of OFDM signal is its high peak-to-average power ratio (PAPR). As OFDM signal is essentially a sum of multiple subcarrier signals aligned in frequency-domain, its probability density function in time-domain resembles Gaussian distribution, and thus its amplitude has high PAPR. The constant amplitude zero auto-correlation (CAZAC) precoding had been proposed as one of PAPR reduction techniques for OFDM signals [5]. Afterwards we had found out that the CAZAC precoding (CAZAC-OFDM) made the PAPR of M-array quadrature amplitude modulation (M-QAM) OFDM signals into the PAPR of M-QAM single-carrier signals [6].Moreover, we had demonstrated the theoretical verification of the CAZAC-OFDM [7]. Here, we also had found out the CAZAC- OFDM accorded the amplitude of IFFT output signal with the amplitude of input DATA and the time ordering of IFFT output signal was unambiguously determined. We verify that this determination is caused by compatibility with the IFFT operation, not by the autocorrelation properties of the CAZAC sequence. Moreover, if we embed null data at a fixed position in time-domain, we will easily detect the symbol timing shift in the receiver side.

The rest of the paper is outlined as follows: Section II contains overview of symbol timing estimation in conventional OFDM system and verifies that time domain signals of OFDM which cannot be manipulated in conventional can be shaped by using CAZAC precoder, and Section III contains description of proposed system using the characteristics of CAZAC-OFDM, and section IV presents performance evaluation and computer simulation results, and section $\mathrm{V}$ concludes the paper.

\section{VISIBLE LIGHT COMMUNICATION AND CAZAC SEQUENCE}

\subsection{Visible Light Communication (VLC)}

Visible light communication has developed rapidly as an attractive technology for supplementing conventional radio frequency communication, which is made possible by the rise of LEDs. One of the important characteristics of the reason why LEDs is used for visible light communication is fast responsiveness.

LEDs are able to follow frequency high enough to send useful data and it is a frequency that is too high to be perceived by the human eye. Therefore, it is possible to complement the role of the LEDs widely used as the lighting and the function as the communication device. 


\subsection{OFDM system}

As well-known, OFDM system enables the limited frequency bandwidth of LEDs or LDs can be fully-used.

In an OFDM transmitter, an inverse fast Fourier transform (IFFT) is performed after primary modulation of a data sequence. As a result, the value after primary modulation is placed on each subcarrier, and a spectrum is formed in which each subcarrier overlaps. Even if each subcarrier overlaps, due to mutual orthogonality, respectively demodulation is possible, so that frequency utilization efficiency is excellent.

The nth sample with the input signal $\boldsymbol{X}$ (length $N$ ) after mapping is defined as

$$
x[n]=\frac{1}{N} \sum_{k=0}^{N-1} X[k] e^{j \frac{2 \pi}{N} k n}
$$

Where $X[k]$ is the frequency-domain signal, and $N$ is the number of subcarrier, $j=\sqrt{-1}$. After IFFT, a guard interval is inserted in the baseband signal. The OFDM symbol is generated by the above procedure.

Since OFDM signals are composed of signals on a symbol-by-symbol basis, when performing FFT for demodulation, it is necessary to find the beginning of the symbol and open the FFT window at an exact timing.

Generally, symbol timing is estimated by using correlation characteristics with known or existing signals [8], [9]. One method is to detect a peak by cross correlation using a preamble. It adds a known sequence to the beginning of the signal and establishes symbol synchronization by calculating the correlation value between the known sequence and the signal.

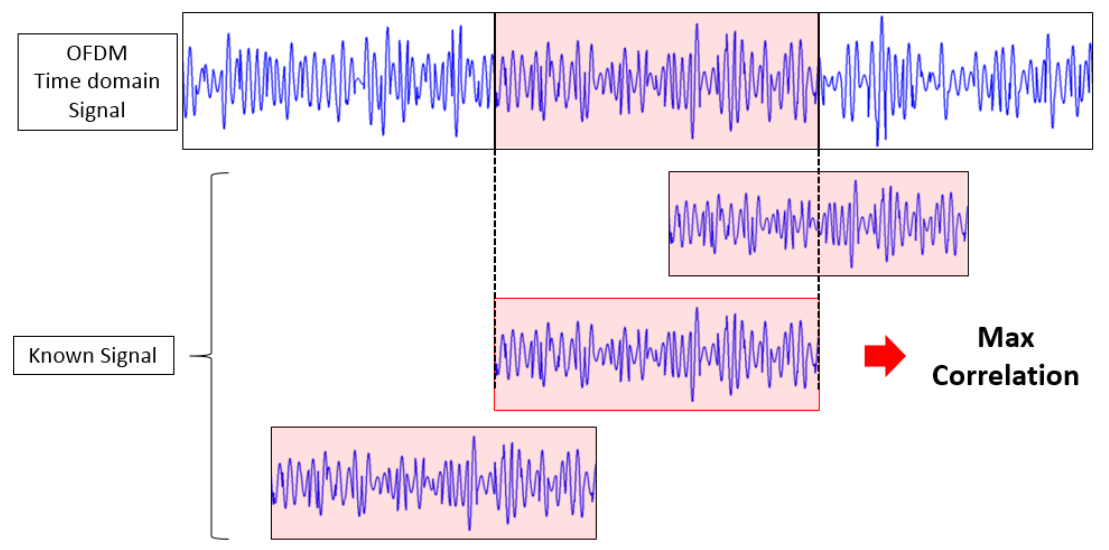

Figure 2.Symbol timing estimation by cross-correlation

When the known sequence matches the signal, the correlation value becomes the maximum, so we use this to detect the beginning of the symbol.

Another, there is estimation using the guard interval. In this case, by adding a guard interval, the same value exists in the portion apart from the copied portion by the original symbol length. In other words, the correlation between the normal waveform and the waveform delayed by the 
original symbol length becomes higher in the copied part. From this, the symbol timing is estimated by detecting the peak of the correlation value.

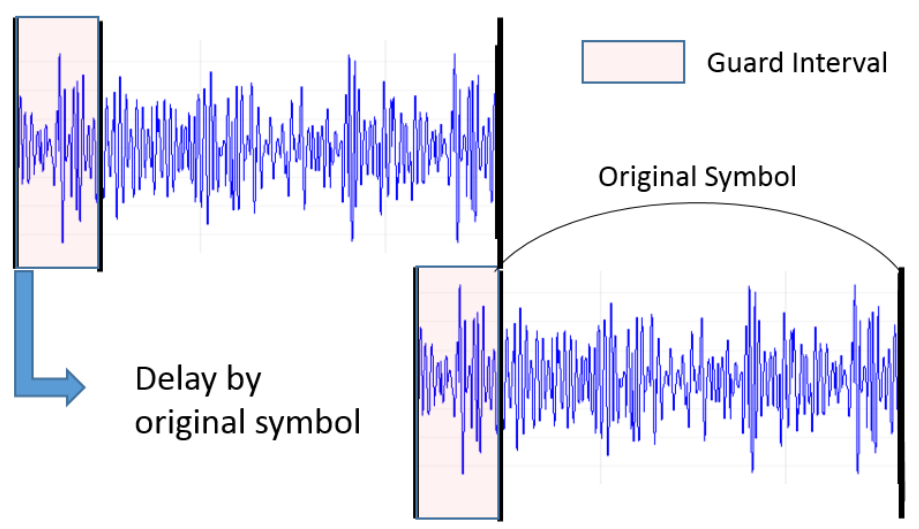

Figure 3. Symbol timing estimation using Guard Interval

\subsection{Effect of Symbol Timing Offset (STO)}

Consider the effect of STO when guard interval to be described later is used. First, FFT is defined as

$$
\mathrm{X}[\mathrm{n}]=\sum_{k=0}^{N-1} x[k] e^{-j \frac{2 \pi}{N} k n}
$$

As can be seen from (1), (2), demodulation at the receiver cannot be demodulated without distortion unless all $0 \sim \mathrm{k}-1$ samples are available. Here, when $\delta$ sample offset occurs in the time domain, assuming that inter-symbol interference does not occur, following as

$$
\mathrm{X}^{\prime}[\mathrm{n}]=\sum_{k=0}^{N-1} x[k+\delta] e^{-j \frac{2 \pi}{N} k n}
$$

Similarly, considering the correspondence of (1), (3), it becomes as follows

$$
\begin{gathered}
\mathrm{X}^{\prime}[\mathrm{n}]=\frac{1}{\mathrm{~N}} \sum_{k=0}^{N-1}\left\{\sum_{m=0}^{N-1} X[m] e^{j \frac{2 \pi}{N} m(n+\delta)}\right\} e^{-j \frac{2 \pi}{N} k n} \\
=\frac{1}{\mathrm{~N}} \sum_{k=0}^{N-1} X[m] e^{j \frac{2 \pi}{N} m \delta} \sum_{k=0}^{N-1} e^{j \frac{2 \pi}{N}(m-k) n} \\
=\mathrm{X}[\mathrm{n}] e^{j \frac{2 \pi}{N} n \delta}
\end{gathered}
$$

From this, it can be seen that the STO within the guard interval generates a phase rotation proportional to the offset after the demodulation. The constellation when setting the FFT window such as Figure.4 is shown as Figure.5. 


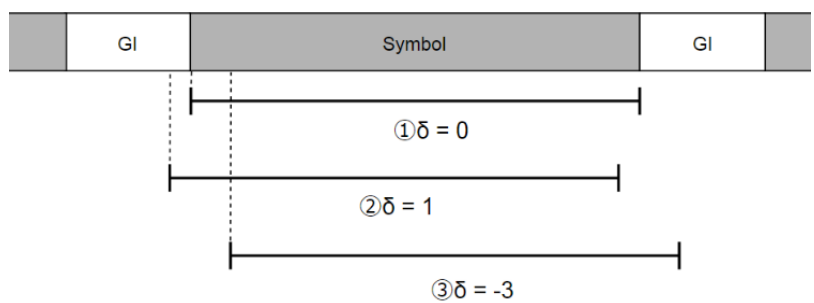

Figure 4.Symbol Timing Offset with OFDM

In the case of (1),the FFT window is set at an exact timing, so there is no offset, and a clean constellation appears after demodulation. In the case of (2), the FFT window is set before the exact point. Due to a guard interval, symbols are retrieved without interfering with adjacent symbols. However, since the extracted samples are cyclically shifted in the correct arrangement, the phase rotation proportional to the offset is added to the demodulated signal as shown in (4).It can be corrected by using the pilot signal in the symbol. In the case of (3), the FFT window is set after the exact point. In this case, it interferes with the adjacent symbol, not only the above-mentioned phase rotation but also distortion in the amplitude direction is added. Therefore, the signal deteriorates.

In conventional OFDM system, it is possible to correctly reproduce by correcting the offset within the guard interval by using the guard interval, but when the guard interval is not used, it is impossible to correct deterioration due to the offset. Therefore, symbol timing needs to be estimate exactly.
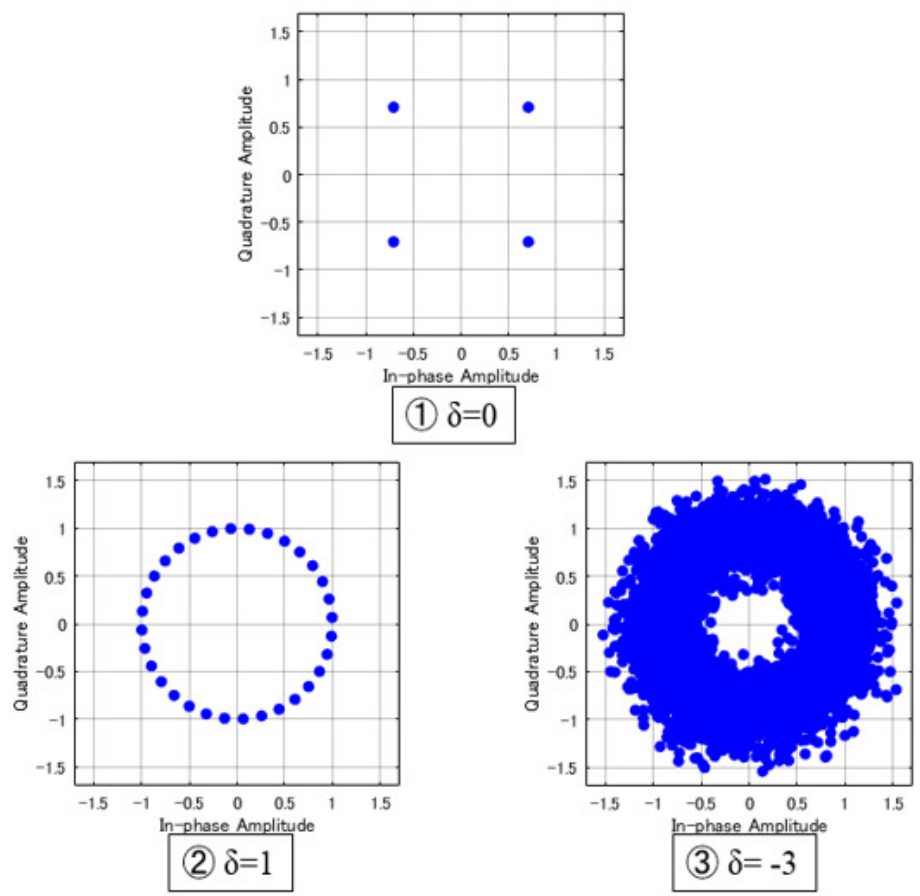

Figure 5. Constellation for (1) (3) Symbol Timing Offset with OFDM (QPSK, FFT size:64) 


\subsection{CAZAC-OFDM}

CAZAC-OFDM is an OFDM method precoded by using the CAZAC sequence shown in equation (5).

$$
\boldsymbol{C}(k)=\left\{\begin{array}{c}
\exp \left(j \frac{\pi u}{N} k^{2}\right) \mathrm{N} \text { is even } \\
\exp \left(j \frac{\pi u}{N} k(k+1)\right) \mathrm{N} \text { is odd } \\
k=0,1, \ldots, N-1
\end{array}\right.
$$

In this case, when CAZAC precoder is applied in OFDM, the signal length $\mathrm{N}$ mostly takes a power of 2 because of FFT. Also, if $u=1$, the Zadoff-Chu sequence used from the formula (5) is as follows.

$$
\boldsymbol{C}(k)=\exp \left(j \frac{\pi}{N} k^{2}\right)(k=0,1, \ldots, N-1)
$$

From (6), an $\mathbf{N} \times \mathrm{N}$ square matrix $\mathbf{M}$ is generated. The matrix equation is defined as

$$
\mathbf{M}=\frac{1}{\sqrt{N}}\left[\begin{array}{cccc}
\boldsymbol{c}_{0} & \boldsymbol{c}_{1} & \cdots & \boldsymbol{c}_{N-1} \\
\boldsymbol{c}_{N} & \boldsymbol{c}_{N+1} & \cdots & \boldsymbol{c}_{2(N-1)} \\
\vdots & \vdots & \ddots & \vdots \\
\boldsymbol{c}_{(N-1) N} & \boldsymbol{c}_{(N-1) N+1} & \ldots & \boldsymbol{c}_{(N-1)^{2}}
\end{array}\right]
$$

In transmitter side, CAZAC precoder signal is generated by multiplying the mapping data by this matrix $\mathbf{M}$. In receiver side, multiply the received symbol $\mathrm{Y}$ in frequency domain and the inverse matrix $\left\{\mathbf{M}^{\mathrm{H}}\right\}^{\mathrm{T}}$ after FFT.

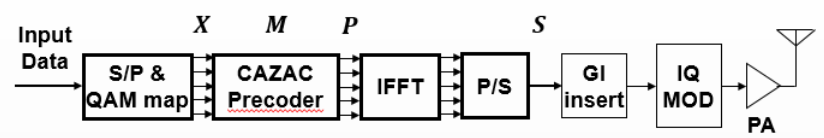

Figure 6. The receiver configuration using CAZAC precoder.

According to Figure.6, $\mathbf{X}$ is QAM Vector, $\mathbf{P}$ is Modulation Vector for IFFT, $\mathbf{M}$ is CAZAC Matrix.Then Sas

$$
\begin{aligned}
& \boldsymbol{S}\left(t_{n}\right)=\frac{1}{N} \sum_{k=0}^{N-1} \boldsymbol{P}_{k} e^{j 2 \pi k n / N} \\
& =\sum_{k=0}^{N-1}\left\{\sum_{m=0}^{N-1} e^{j \pi(m+k N)^{2} / L} \boldsymbol{X}_{m}\right\} e^{j 2 \pi k n / N} \\
& =\sum_{m=0}^{N-1} e^{\frac{j \pi m^{2}}{N^{2}}} \boldsymbol{X}_{m}\left\{\sum_{k=0}^{N-1} e^{\frac{j 2 \pi k(m+n)}{N}} e^{j \pi k^{2}}\right\}
\end{aligned}
$$

Where $k$ is an integer not less than 0 , so the following equation is developed.

$$
\exp \left(j \pi k^{2}\right)=\left\{\begin{array}{cc}
1 & (k: \text { even }) \\
-1 & (k: \text { odd })
\end{array}\right.
$$

To lead the equation (10) from the equation (9). 


$$
\exp \left(j \pi k^{2}\right)=(-1)^{k}
$$

Substituting the equation (10) into the equation (8) lead to the equation (11).

$$
\boldsymbol{S}\left(t_{n}\right)=\sum_{m=0}^{N-1} e^{j \pi m^{2} / N^{2}} \boldsymbol{X}_{m}\left\{\sum_{k=0}^{N-1}\left\{-e^{j 2 \pi(m+n) / N}\right\}^{k}\right\}
$$

The inside of \{\} in equation (11) is the sum of the geometric progression. Therefore, equation (12) is derived.

$$
\sum_{k=0}^{N-1}\left\{-e^{j 2 \pi(m+n) / N}\right\}^{k}=\left\{\begin{array}{l}
N\left(-e^{j 2 \pi(m+n) / N}=1\right) \\
0\left(-e^{j 2 \pi(m+n) / N} \neq 1\right)
\end{array}\right.
$$

When $2 \pi(m+n) / N=1,2(m+n) / N$ is an integer and odd number. Also, $\mathrm{n}$ and $m$ are $0 \leq n \leq N-1,0 \leq m \leq N-1$. Equation (13) follows on account of these relationships.

$$
m=\frac{N}{2}-n(\bmod N)
$$

Equation (14) is derived from equations (11), (12) and (13).

$$
\boldsymbol{S}\left(t_{n}\right)=\boldsymbol{X}\left(\left(\frac{N}{2}-n\right)_{\bmod N}\right) \boldsymbol{C}\left(\left(\frac{N}{2}-n\right)_{\bmod N}\right)
$$

From the above, we have theoretically derived $\mathbf{S}\left(\mathrm{t}_{\mathrm{n}}\right)$ for arbitrary $\mathrm{n}$. The time waveform of CAZAC-OFDM is obtained by phase-rotating the value after mapping.
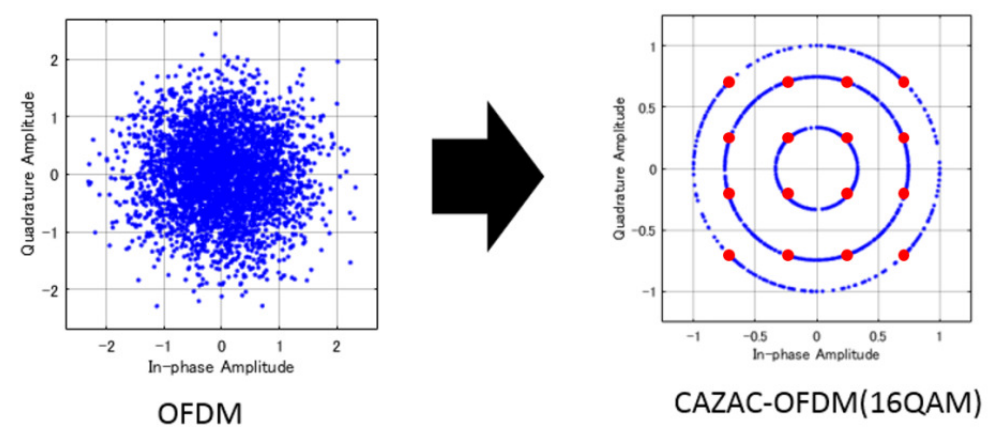

Figure7. Time domain Signals with CAZAC precoder influenced by the value after mapping.

Here, concrete calculation in the case of $\mathrm{N}=4$ is performed to understand well. According to Figure.6, $\mathbf{P}=\mathbf{M X}$ is obvious. Therefore, $\mathbf{P}$ as

$$
\left[\begin{array}{l}
\boldsymbol{P}_{0} \\
\boldsymbol{P}_{1} \\
\boldsymbol{P}_{2} \\
\boldsymbol{P}_{3}
\end{array}\right]=\left[\begin{array}{cccc}
1 & e^{j \frac{\pi}{16}} & e^{j \frac{\pi}{4}} & e^{j \frac{9 \pi}{16}} \\
-1 & -e^{j \frac{\pi}{16}} & e^{j \frac{\pi}{4}} & -e^{j \frac{\pi}{16}} \\
1 & -e^{j \frac{\pi}{16}} & e^{j \frac{\pi}{4}} & -e^{j \frac{9 \pi}{16}} \\
-1 & e^{j \frac{\pi}{16}} & e^{j \frac{\pi}{4}} & e^{j \frac{\pi}{16}}
\end{array}\right]\left[\begin{array}{l}
\boldsymbol{X}_{0} \\
\boldsymbol{X}_{1} \\
\boldsymbol{X}_{2} \\
\boldsymbol{X}_{3}
\end{array}\right]
$$




$$
=\left[\begin{array}{c}
\boldsymbol{X}_{0}+e^{j \frac{\pi}{16}} \boldsymbol{X}_{1}+e^{j \frac{\pi}{4}} \boldsymbol{X}_{2}+e^{j \frac{9 \pi}{16}} \boldsymbol{X}_{3} \\
-\boldsymbol{X}_{0}-e^{j \frac{9 \pi}{16}} \boldsymbol{X}_{1}+e^{j \frac{\pi}{4}} \boldsymbol{X}_{2}-e^{j \frac{\pi}{16}} \boldsymbol{X}_{3} \\
\boldsymbol{X}_{0}-e^{j \frac{\pi}{16}} \boldsymbol{X}_{1}+e^{j \frac{\pi}{4}} \boldsymbol{X}_{2}-e^{j \frac{\pi}{16}} \boldsymbol{X}_{3} \\
-\boldsymbol{X}_{0}+e^{j \frac{9 \pi}{16}} \boldsymbol{X}_{1}+e^{j \frac{\pi}{4}} \boldsymbol{X}_{2}+e^{j \frac{\pi}{16}} \boldsymbol{X}_{3}
\end{array}\right]
$$

$\mathbf{S}$ is the IFFT-operated signal of $\mathbf{M}$. Therefore $\mathbf{P}_{0} \sim \mathbf{P}_{3}$ is respectively multiplied by following phase-rotation value for subcarriers of $f_{0}, 2 f_{0}, 3 f_{0}, 4 f_{0}$ and summed.

$$
\begin{aligned}
& \mathrm{e}^{\mathrm{j} \frac{2 \pi(\mathrm{n}+1)}{4} \mathrm{t}_{\mathrm{m}} \quad(\mathrm{n}=0,1,2,3)} \\
& \mathrm{t}_{\mathrm{m}} \text { : IFFT output time order }
\end{aligned}
$$

$$
\left(t_{m}=0,1,2,3\right)
$$

First, calculate $\mathbf{S}\left(\mathrm{t}_{0}\right)$.

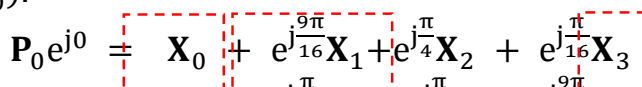

$$
\begin{aligned}
& \mathbf{P}_{1} \mathrm{e}^{\mathrm{j} 0}=-\mathbf{X}_{0}^{0}-\mathrm{e}^{\mathrm{j} \frac{\pi}{16}} \mathbf{X}_{1}+\mathrm{e}^{\mathrm{j} \frac{\pi}{4}} \mathbf{X}_{2}-\mathrm{e}^{\mathrm{j} \frac{9 \pi}{16}} \mathbf{X}_{3}
\end{aligned}
$$

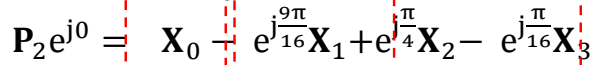

$$
\begin{aligned}
& \mathbf{P}_{3} \mathrm{e}^{\mathrm{j} 0}-\mathbf{X}_{0}+\mathrm{e}^{\mathrm{j} \frac{\pi}{16}} \mathbf{X}_{1}+\mathrm{e}^{\mathrm{j} \frac{\pi}{4}} \mathbf{X}_{2}+\mathrm{e}^{\mathrm{j} \frac{\pi}{16} \mathbf{X}_{3}} \\
& \begin{array}{r:r:r} 
& 0 & 00
\end{array} \\
& \mathbf{S}\left(\mathrm{t}_{2}\right)=\mathbf{P}_{0} \mathrm{e}^{\mathrm{j} 0}+\mathbf{P}_{1} \mathrm{e}^{\mathrm{j} 0}+\mathbf{P}_{2} \mathrm{e}^{\mathrm{j} 0}+\mathbf{P}_{3} \mathrm{e}^{\mathrm{j} 0} \\
& =4 \mathrm{e}^{\mathrm{j} \frac{\pi}{4}} \mathbf{X}_{2}=4 \boldsymbol{C}_{2} \boldsymbol{X}_{2} \\
& \left(\boldsymbol{c}_{k}=e^{\frac{j \pi k^{2}}{16}}\right)
\end{aligned}
$$

Next, calculate $\mathbf{S}\left(\mathrm{t}_{1}\right)$.

$$
\begin{aligned}
& \boldsymbol{P}_{0} e^{j \frac{\pi}{2}}=e^{j \frac{\pi}{2}} \boldsymbol{X}_{0}+e^{j \frac{\pi}{16}} \boldsymbol{X}_{1}+e^{j \frac{3 \pi}{4}} \boldsymbol{X}_{2} e^{j \frac{\pi}{16}} \boldsymbol{X}_{3} \\
& \boldsymbol{P}_{1} e^{j \pi}=\quad \boldsymbol{X}_{0}+e^{j \frac{\pi}{16}} \boldsymbol{X}_{1}-e^{j \frac{\pi}{4}} \boldsymbol{X}_{2}+e^{j \frac{9 \pi}{16}} \boldsymbol{X}_{3} \\
& \boldsymbol{P}_{2} e^{j \frac{3 \pi}{2}}=e^{j \frac{\pi}{2}} \boldsymbol{X}_{0}+e^{j \frac{\pi}{16} \boldsymbol{X}_{1}}-e^{j \frac{3 \pi}{4}} \boldsymbol{X}_{2}-e^{j \frac{9 \pi}{16}} \boldsymbol{X}_{3}
\end{aligned}
$$

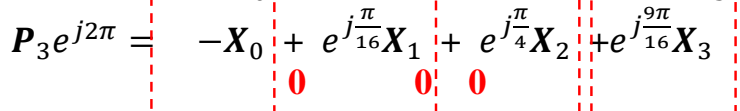

$$
\begin{aligned}
& \mathbf{S}\left(\mathrm{t}_{1}\right)=\mathbf{P}_{0} \mathrm{e}^{\mathrm{j} \frac{\pi}{2}}+\mathbf{P}_{1} \mathrm{e}^{\mathrm{j} \pi}+\mathbf{P}_{2} \mathrm{e}^{\mathrm{j} \frac{3 \pi}{2}}+\mathbf{P}_{3} \mathrm{e}^{\mathrm{j} 2 \pi} \\
& =4 e^{j \frac{\pi}{16}} \boldsymbol{X}_{1}=4 \boldsymbol{C}_{1} \boldsymbol{X}_{1} \\
& \left(\boldsymbol{c}_{k}=e^{\frac{j \pi k^{2}}{16}}\right)
\end{aligned}
$$

Similarly, about $\mathbf{S}\left(\mathrm{t}_{2}\right)$ and $\mathbf{S}\left(\mathrm{t}_{3}\right)$,

$$
\begin{gathered}
\mathbf{S}\left(\mathrm{t}_{2}\right)=\mathbf{P}_{0} e^{j \pi}+\mathbf{P}_{1} \mathrm{e}^{\mathrm{j} 2 \pi}+\mathbf{P}_{2} e^{j 3 \pi}+\mathbf{P}_{3} \mathrm{e}^{\mathrm{j} 4 \pi} \\
=4 \boldsymbol{X}_{0}=4 \boldsymbol{C}_{0} \boldsymbol{X}_{0} \\
\mathbf{S}\left(\mathrm{t}_{3}\right)=\mathbf{P}_{0} \mathrm{e}^{j \frac{3 \pi}{2}}+\mathbf{P}_{1} \mathrm{e}^{\mathrm{j} 3 \pi}+\mathbf{P}_{2} \mathrm{e}^{\mathrm{j} \frac{9 \pi}{2}}+\mathbf{P}_{3} \mathrm{e}^{\mathrm{j} 6 \pi} \\
=4 e^{j \frac{\pi}{16}} \boldsymbol{X}_{3}=4 \boldsymbol{C}_{3} \boldsymbol{X}_{3}
\end{gathered}
$$

As a result, 


$$
\left[\begin{array}{l}
\mathbf{S}\left(\mathrm{t}_{0}\right) \\
\mathbf{S}\left(\mathrm{t}_{1}\right) \\
\mathbf{S}\left(\mathrm{t}_{2}\right) \\
\mathbf{S}\left(\mathrm{t}_{3}\right)
\end{array}\right]=\left[\begin{array}{l}
\boldsymbol{C}_{2} \boldsymbol{X}_{2} \\
\boldsymbol{C}_{1} \boldsymbol{X}_{1} \\
\boldsymbol{C}_{0} \boldsymbol{X}_{0} \\
\boldsymbol{C}_{3} \boldsymbol{X}_{3}
\end{array}\right]
$$

(Ignore the coefficients as they are not important.)

$$
\begin{aligned}
& \left|\boldsymbol{S}\left(t_{n}\right)\right|=\left|\boldsymbol{X}_{\left(\frac{N}{2}-n\right) \bmod N}\right| \\
& \quad \text { because of }\left|\boldsymbol{C}_{\left(\frac{N}{2}-n\right) \bmod N}\right|=1
\end{aligned}
$$

Computation is complicated, but many terms cancel clearly each other, resulting in the phase-rotating value after mapping remaining. In sum, we found an astonishing fact, that is the CAZAC precoding makes the amplitudes of M-QAM OFDM signals into the amplitudes of M-QAM single-carrier signals.

\section{PROPOSED SYSTEM}

As has been noted, CAZAC precoding can make the amplitudes of M-QAM OFDM signals into the amplitudes of M-QAM single-carrier signals. As one application example that can use this characteristic of the waveform of CAZAC-OFDM, we propose inserting a null at a fixed position of the symbol of the time waveform and estimate the symbol timing by detecting the null at the receiving side.

Figure.8 schematically shows the relationship between before and after modulation of CAZAC-OFDM in transmitter side when FFT size is 64.It is arranged in the order of $\mathbf{S}\left(\mathrm{t}_{0}\right)=\mathbf{X}$ (32) $\mathbf{C}(32), \mathbf{S}\left(\mathrm{t}_{1}\right)=\mathbf{X}(31) \mathbf{C}(31), \ldots, \mathbf{S}\left(\mathrm{t}_{32}\right)=\mathbf{X}(0) \mathbf{C}(0), \mathbf{S}\left(\mathrm{t}_{33}\right)=\mathbf{X}(63) \mathbf{C}(63), \ldots, \mathbf{S}\left(\mathrm{t}_{63}\right)=\mathbf{X}(33) \mathbf{C}(33)$ from the beginning of the symbol. This time 2 nulls are embedded at the end of the symbol.

Therefore, the values of $\mathbf{X}(33), \mathbf{X}(34)$ are fixed to 0 , and then the transmission waveform of CAZAC-OFDM is generated.Figure9. shows the amplitudes of time domain signals of CAZAC-OFDM (Mapping: QPSK) by the method described above. It is apparent that nulls are inserted firmly at the end of the symbol.

The receiving side judges null and detects the head by the following block configuration(Figure.10). Based on the value obtained by amplitude estimation, the threshold value is set to be half of the distance to the data point closest to 0 , using the fact that the transmission waveforms of CAZAC-OFDM are constant amplitudes. 


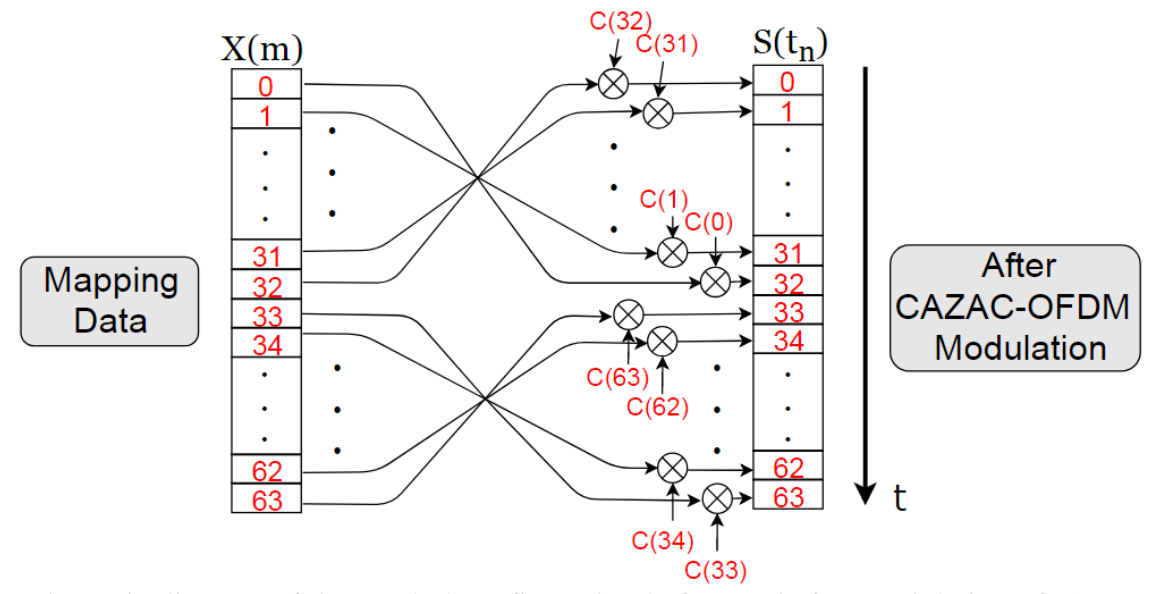

Figure 8. Schematic diagram of the symbol configuration before and after modulation of CAZAC-OFDM (64FFT)

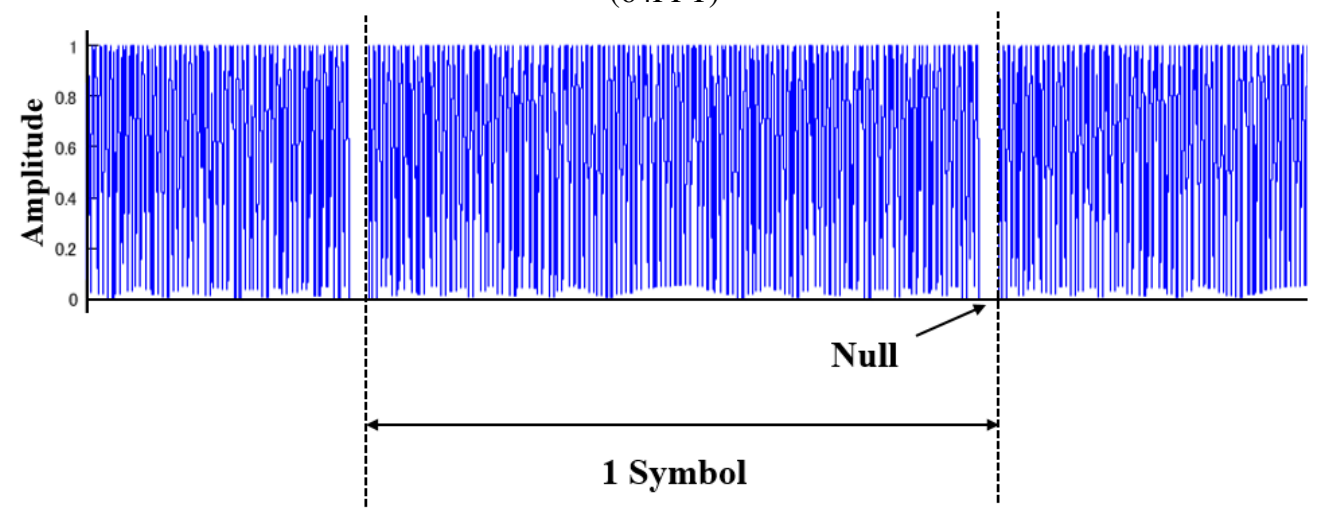

Figure 9. The amplitudes of time domain signals of CAZAC-OFDM embedded Nulls(QPSK)

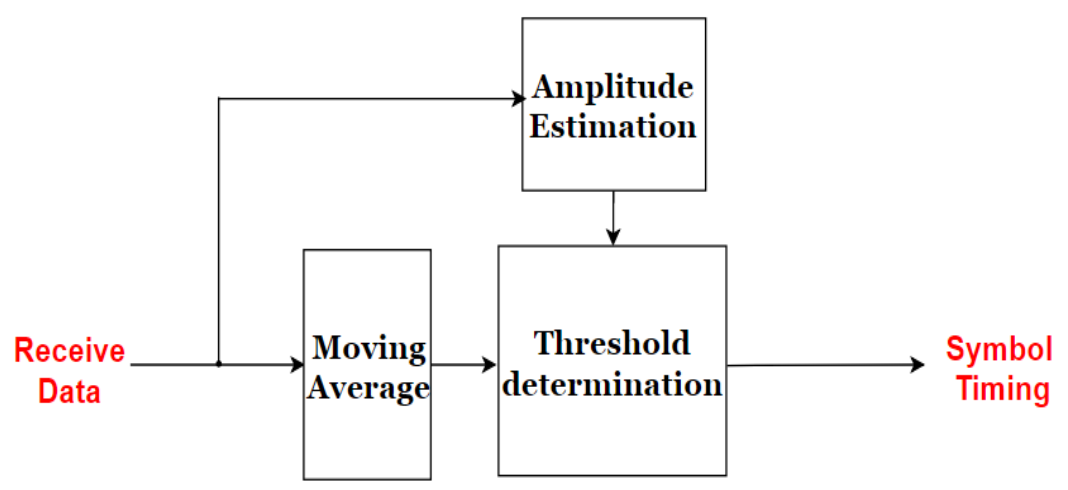

Figure 10.The block configuration of null detection. 

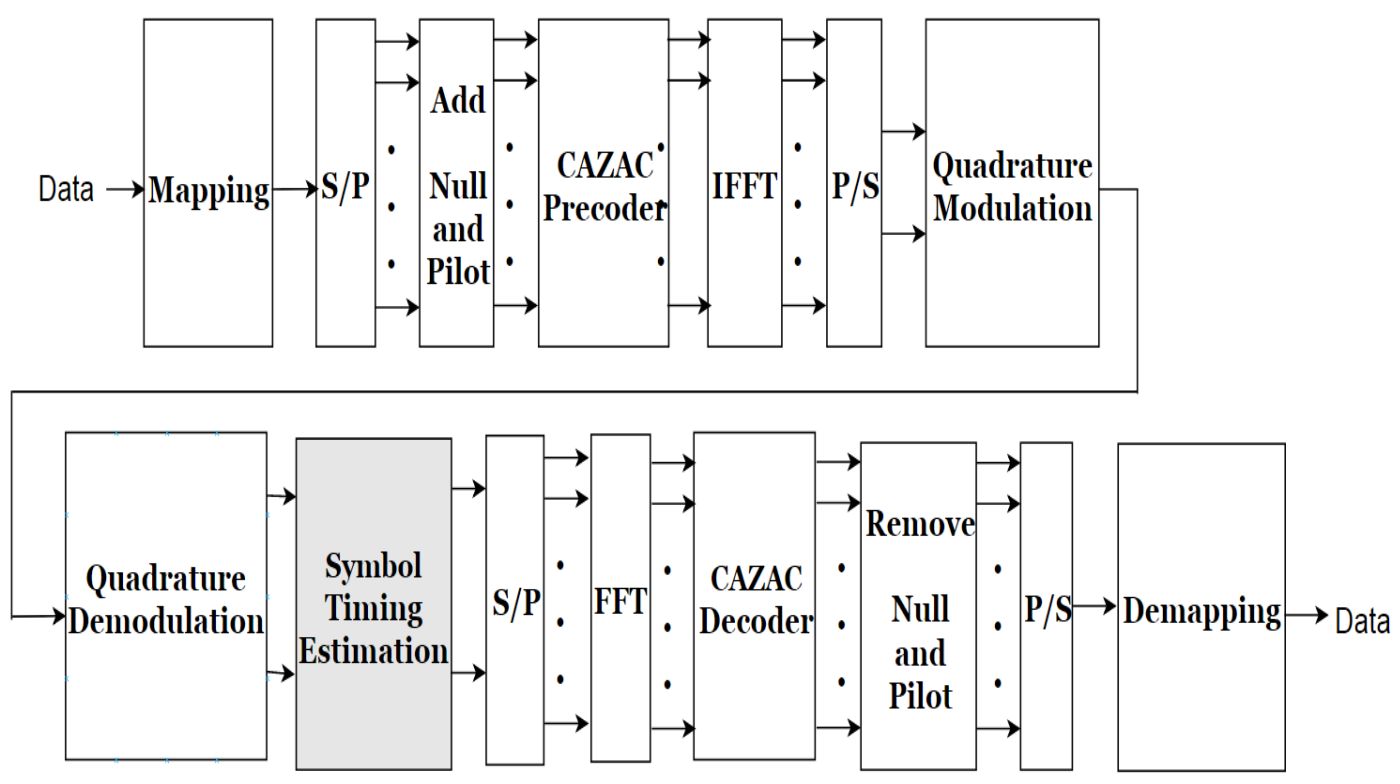

Figure 11. The proposed CAZAC-OFDM system

\section{SIMULATION RESULTS AND DISCUSSIONS}

\subsection{Simulation Results}

To evaluate the performance of the proposed method, simulation was performed according to the specifications in Table 1. The simulation is performed in MATLAB using communications system toolbox. In addition, head detection using correlation characteristics by guard interval was used as a comparison target. Since the proposed method inserts nulls, it is considered necessary to consider noise. Therefore, among the range where the data itself can demodulate error free in consideration of error correction in this environment $\left(\mathrm{BER}<10^{-3}\right.$ ), comparison is made at SNR of the worst noise of $15 \mathrm{~dB}$.

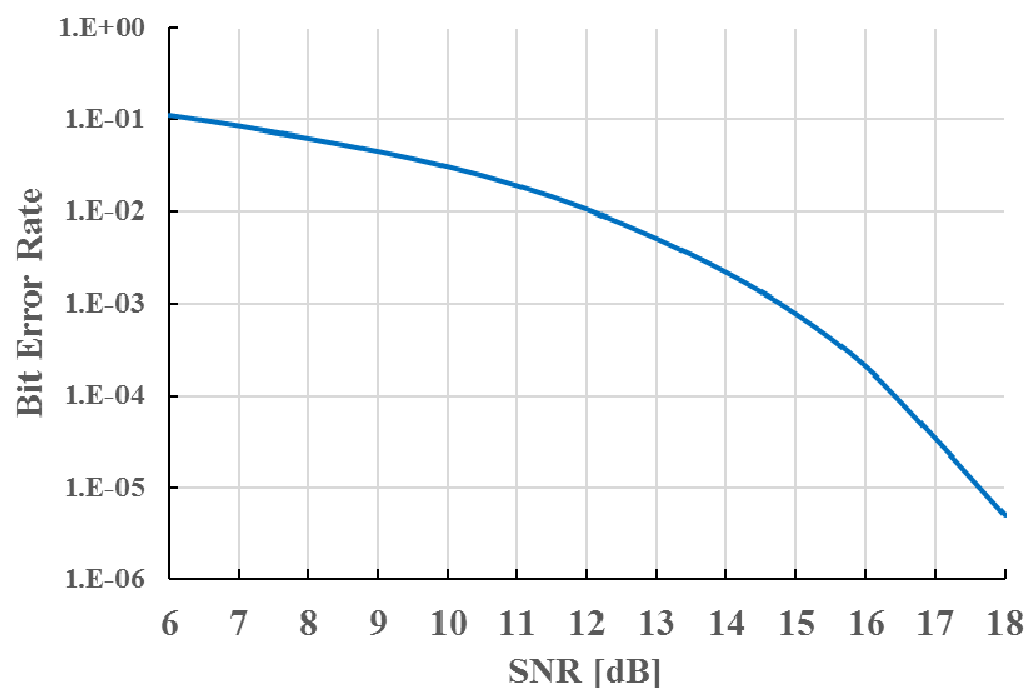

Figure12. BER of simulation work environment. 
TABLE 1. SPECIFICATION FOR SIMULATION

\begin{tabular}{|c|c|c|}
\hline & $\begin{array}{c}\text { Auto-Correlation } \\
\text { (GI) }\end{array}$ & Proposal \\
\hline Mapping & \multicolumn{2}{|c|}{ 16QAM } \\
\hline $\begin{array}{c}\text { Number of } \\
\text { Data subcarriers }\end{array}$ & 59 & 61 \\
\hline $\begin{array}{c}\text { Number of } \\
\text { Pilot subcarriers }\end{array}$ & 1 & 1 \\
\hline Number of \\
Null
\end{tabular}

Figure 13 shows the comparison of detection accuracy with each method. The vertical axis is the timing metric of each method. 0 on the horizontal axis represents the first sample of the symbol. The largest peak appears near 0 in both methods. However, compared with the method using GI, the proposed method shows that a sharp peak appears. From this, it is apparent that the proposed method achieves highly accurate head detection compared to the case of head detection by correlation using GI. In this simulation, 2 samples per symbol were used as synchronization nulls. The throughput at this time is about $30 \%$ higher than the throughput when the guard interval of $1 / 4$ symbol length is used.

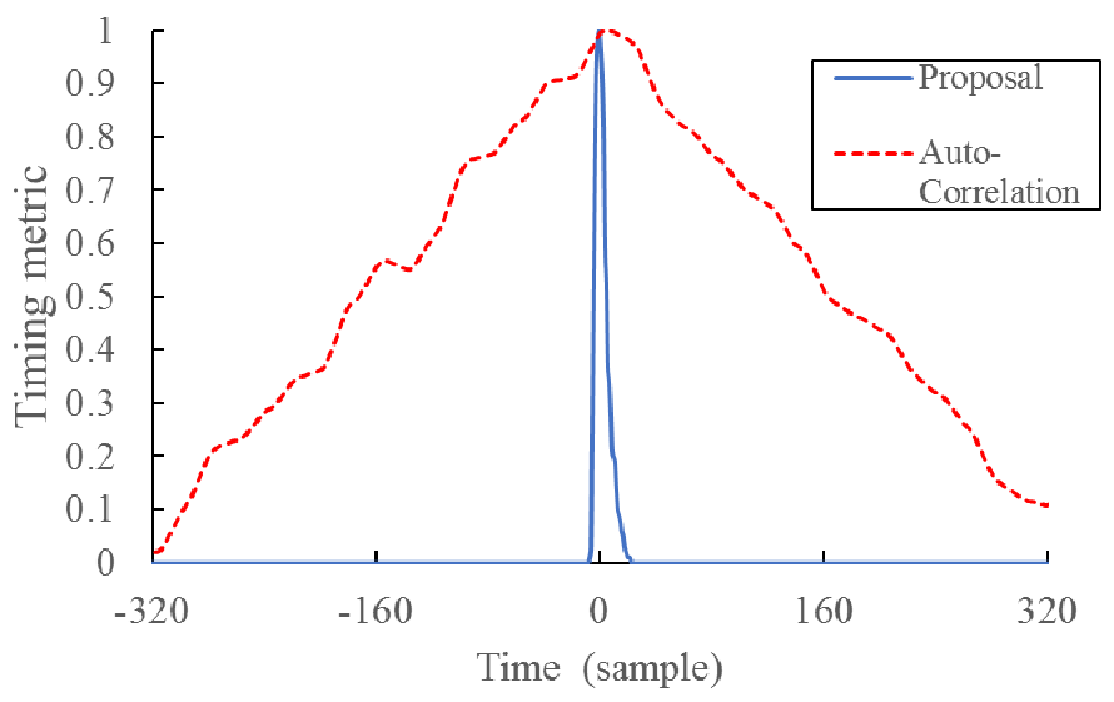

Figure 13. Comparison of detection accuracy. 


\section{CONCLUSION}

In this paper, we have demonstrated that it is possible by using CAZAC-OFDM to make the amplitudes of M-QAM OFDM signals into the amplitudes of M-QAM single-carrier signals. We verified that the OFDM time domain signals can be shaped by using CAZAC precoder. We also verified that, as one application example that can use the characteristic of CAZAC precoder, a new technique of symbol timing estimation provided sufficient performance.

\section{REFERENCES}

[1] A. Jovicic, J. Li, T. Richardson" Visible light communication: opportunities, challenges and the path to market", IEEE Communications Magazine, pp.26-32, Dec. 2013

[2] H. Elgala, R. Mesleh, H. Haas:'Indoor broadcasting via white LEDs and OFDM", IEEE Transactions on Consumer Electronics, pp.1127-1134, Aug. 2009

[3] G. Cossu, A. M. Khalid, P. Choudhury, R. Corsini, E. Ciaramella, "3.4 Gbit/s visible optical wireless transmission based on RGB LED", Opt. Exp., pp. B501-B506, Dec. 2012

[4] Z Feng, M Tang, S Fu, L Deng, Q Wu, R Lin, R Wang, "Performance-enhanced direct detection optical OFDM transmission with CAZAC equalization, " IEEE Photonics Technology Letters, pp. 1507-1510,July 2015

[5] I. Baig and V. Jeoti, "PAPR Reduction in OFDM Systems: Zadoff-Chu Matrix Transform Based Pre/Post-Coding Techniques," in Proc.of the 2nd International Conference on Computational Intelligence, Communication Systems and Networks, pp. 373-377, July 2010.

[6] R. Ishioka, T. Kimura and M. Muraguchi, "A Proposal for a New OFDM Wireless System using a CAZAC Precoding Scheme,”Proc. AICT 2017, pp. 47-51, June 2017.

[7] Kazuki Miyazawa, Tomotaka Kimura, Masahiro Muraguchi, "Proposal of visible light OFDM system with CAZAC equalization," 23rd Asia-Pacific Conference on Communications (APCC), pp.491-496, Dec. 2017.

[8] T.M. Schmidl, D.C. Cox:" Robust frequency and timing synchronization for OFDM", IEEE Transactions on Communications, pp.1613-1621, Dec. 1997

[9] B.Park, H.Cheon, C. Kang, D. Hong:"A novel timing estimation method for OFDM systems", IEEE Communications Latters, pp. 239-241, May 2003 